discovery of the Higgs boson by the LHC at CERN, Europe's particlephysics laboratory near Geneva, Switzerland.

But physicists know that the standard model is not the end of the story, not least because it doesn't include gravity or explain dark matter. It is at best part of a wider undiscovered framework, or an approximation of a more fundamental theory. At the optimistic start-up of the LHC in 2009, the path beyond the standard model seemed marked out. Many theorists assumed that particles predicted by an elegant extension to the model - supersymmetry - would reveal themselves as soon as colliders could reach a high enough energy.

Since then, LHC experiments have smashed together quadrillions of particles at record energies, and no supersymmetric particles have emerged. Proponents have not given up hope, but they have ruled out the simplest versions of their theories. Now, rather than rely on conjuring new particles, many are hoping to observe their effects indirectly, prying open tiny cracks in the standard model by exploring a handful of findings that don't quite fit predictions. Two reviews in this week's Nature examine one area in which anomalies are accruing (see pages 221 and 227). Excitement is growing about the strange and subtle behaviour of particles called $B$ mesons, whose properties make them ripe for observing the effects of new physics.

CERN's LHCb and other ' $B$-factory' experiments, such as Belle at Japan's High Energy Accelerator Research Organization (KEK) in Tsukuba, have often worked in the shadows of their more famous sister experiments. Eschewing the need for higher and higher energies, $B$ factories hoover up data on rare decays of $B$ mesons, which contain $b$ quarks (denoting 'beauty' or 'bottom'). Physicists make precise measurements of how $B$ mesons decay into known particles, searching for tiny discrepancies that would reveal the influence of hypothetical heavy particles that appear fleetingly but leave their mark.

Several such discrepancies exist in various $B$-meson decays, any of which on its own would not raise an eyebrow. But together they seem to fit into a broader picture. Each anomaly hints at the existence of the same

exotic new particle. Its identity is not yet known, but candidates include a heavier type of $Z$ boson - a $Z$ ' - or a 'leptoquark'. Some see this as a wishful cobbling together of statistical flukes: most of the discrepancies are just a few standard deviations away from expectations, and far from the five standard deviations that physicists require to declare a discovery. Yet the strongest of the anomalies - in the rates at which $B$ mesons turn into pairs of different kinds of lepton (electrons and their heavier cous-

"Rather than rely on conjuring new particles, many hope to pry open cracks in the standard model." ins, muons and taus) - has been seen by three separate experiments, whose combined data give a tantalizing standard deviation of four.

Physicists have found few other anomalies to lead them beyond standard-model physics. One involves looking at a deviation in how muons wobble on their axes as they travel in a magnetic field. Many experiments are probing neutrinos - ghostly particles that the standard model assumed were massless, but turned out not to be, and whose basic behaviour physicists are just starting to understand. Meanwhile, other once-promising leads are fading. The proton-radius puzzle - in which experimental physicists disagree over the ubiquitous particle's size by as much as $4 \%$ - could well be down to an incomplete understanding of the measurement process. And a shortage in the number of antineutrinos arriving at experiments near nuclear reactors looks increasingly likely to be caused by errors in calculating the predicted rate.

Beauty physics offers a rare glimmer of hope. The LHCb will continue to rack up data, and KEK's upgraded $B$-factory detector, Belle II, starts up next year. If anomalies fade with more data, a planned upgrade to the LHC - which will ramp up the intensity of collisions from 2025 - could provide a new proving ground.

Discovery in high-energy physics is turning out to be less like swashbuckling through Terra Incognita than some might have hoped. But groping for tiny cracks can also reveal unknown territory - it just takes more patience.

\section{Rescue remedy}

\section{Life-saving treatments for diabetes could come from new work on solving receptor structures.}

$\mathrm{M}$ ost people know about mouth-to-mouth resuscitation and chest compressions, even if they have never had to put them into practice. Some could probably attempt the Heimlich manoeuvre. But how many have heard of glucagon rescue?

To a person with diabetes who has dangerously low blood sugar, a shot of the hormone glucagon delivered by someone else is their best chance of survival. Many carry a life-saving glucagon rescue kit. But it's difficult to use in a hurry. A powder must be mixed into solution, shaken and then delivered with a hypodermic syringe. Even people who are practised struggle. To those who are unfamiliar, the lengthy instructions can be enough to stop them from trying. If a pupil falls into a hypoglycaemic coma, teachers in some US schools have it written into their contracts that they are to call the emergency services instead.

So research that could lead to a better way to address hypoglycaemia and other complications of diabetes is an urgent pursuit. This week, Nature publishes four papers that describe such work. At first glance, the research might seem far removed from the medical front line. It's hardcore structural biology: solved crystal and cryo-electron microscopy (cryo-EM) structures of G-protein-coupled receptors. But the insights could improve glucagon rescue, and lead to other treatments for diabetes and obesity.

In the body, glucagon triggers the conversion of stored glycogen to glucose - the opposite effect to insulin. Stable synthetic insulin is routine in medicine, but glucagon poses more of a problem. Solutions of it go off, hence the tricky rescue kits. Research that could make glucagon solutions stable and rescue kits simpler would have a life-saving impact. How to achieve that? The hormone increases glucose levels by triggering a biochemical sequence that is heavily dependent on a signalling protein. A related signalling protein activates insulin to mop up excess glucose. Type 2 diabetes causes problems because it disrupts this balance. The full structure of these signalling proteins was unknown - until now.

The two proteins are glucagon-like peptide-1 receptor (GLP-1R) and glucagon receptor (GCGR). Their activation and deactivation have opposing roles in glucose homeostasis and insulin release, and so help to regulate metabolism and appetite. They are activated by peptides, which could be adapted as potential drugs. Design of peptide or small-molecule drugs requires data on how and where the molecules bind - information now revealed in the structures. Indeed, in one of the studies, researchers designed a new peptide molecule that activates GLP-1R in mice (A. Jazayeri et al. Nature 546, 254-258; 2017).

Another describes the use of cryo-EM to solve the GLP-1R activated structure in complex with its $\mathrm{G}$ protein signalling partner (Y. Zhang et al. Nature 546, 248-253;2017). It shows how the receptor grabs and secures the peptide, then twists to pass on the signal. Finally, two others report the structures of GLP-1R and GCGR in their inactive forms - both deactivated by small molecules that bind to a different site from the natural hormone (G. Song et al. Nature 546, 312-315 (2017) and H. Zhang et al. Nature 546, 259-264 (2017)). Because small-molecule drugs are easier to design than peptide-hormone mimics, these structures suggest new therapeutic opportunities for regulating glucose homeostasis.

The need is great: in trials, even parents trained to use the kits on their children took an average of 2.5 minutes to mix and deliver glucagon. And many injected nothing but fresh air. They need a helping hand. Structural biology is trying to provide just that. 